\title{
Rare and Exotic Processes at CDF
}

\author{
Ray Culbertson \\ for the CDF Collaboration
}

Fermi National Accelerator Laboratory

\begin{abstract}
We report recent results in CDF searches for rare and exotic processes. In a signaturebased search, we examine the diphoton dataset for additional energetic objects. In a second signature-based search, we search for anomalous production of a photon, a $b$-tagged jet, and missing $E_{T}$. Finally, we search for a Fermiophobic Higgs in the two-photon decay mode, and conclude this Higgs must have mass greater than $106 \mathrm{GeV} / c^{2}$, at $95 \%$ confidence level.
\end{abstract}

Keywords: CDF,Tevatron,photon,diphoton,search,Fermiophobic Higgs

PACS: $13.85 . \mathrm{Rm}$ 14.80.Cp

\section{INTRODUCTION}

Searches at CDF may be broad signature-based searches (SBS) of a dataset for any sign of anomalous production or may be focused on a specific hypothetical model of new physics. In the SBS approach, event counts and kinematics of a dataset are compared to standard model (SM) predictions. This method proceeds quickly, is unprejudiced, and covers many new-physics possibilities. The model-based search can be highly optimized for a particular model, and provide precise limits on that model. Below we present results in two signature-based searches and one using a model-based approach.

\section{SEARCH FOR DIPHOTON AND X EVENTS}

We define a base sample of events with two isolated photons in the central detector $(|\eta|<1.1)$ with $E_{T}>13 \mathrm{GeV}$. We then search for events with additional electrons (central or forward) with $E_{T}>20 \mathrm{GeV}$, in $1.0 \mathrm{fb}^{-1}$. We find three events with an expected background of $6.82 \pm 0.75$. The background is dominated by $Z \gamma$ production, with an electron being misidentified as a photon. This is estimated by defining a sample of events with two electrons and one photon, then applying a probability (derived in data) for an electron to be misidentified as a photon.

In the muon channel $\left(P_{T}>20 \mathrm{GeV}\right.$, in detectors with $|\eta|<1.0$, in $\left.1.0 \mathrm{fb}^{-1}\right)$ we observe no events, and expect $0.79 \pm 0.11$ events, predominately from production of a $W$ or $Z$ with two real photons.

The $\gamma \gamma \tau$ subsample $\left(2.0 \mathrm{fb}^{-1}\right)$ adds the requirement of a hadronically-decaying tau with visible $E_{T}>15 \mathrm{GeV}$. We observe 40 events, with $46 \pm 10$ expected. The background from $W$ or $Z$ is small, so the background from jets misidentified as taus is dominate. We estimate this source by applying a fake rate (derived in data) to events with a loose tau selection. 
For the missing $E_{T}\left(\mathbb{E}_{T}\right)$ subsample, we add a selection on $\mathbb{E}_{T}$ significance, a variable which quantifies how significant the missing $E_{T}$ is based on the kinematics of the event. Primarily, if jet energy is present, missing $E_{T}$ may be produced by fluctuations and therefore an observation of $\mathbb{E}_{T}$ is less significant. For a cut on $\mathbb{E}_{T}$ significance which reduces the mismeasured-energy background by a factor of $10^{5}$, the sample becomes dominated by $W \gamma$ production, where the electron is misidentified as a photon. This background is estimated from Monte Carlo normalized to data. We find 23 events and an expectation of $27.3 \pm 2.3$ events. Figure 1 and 2 show some kinematic distributions from the subsamples.
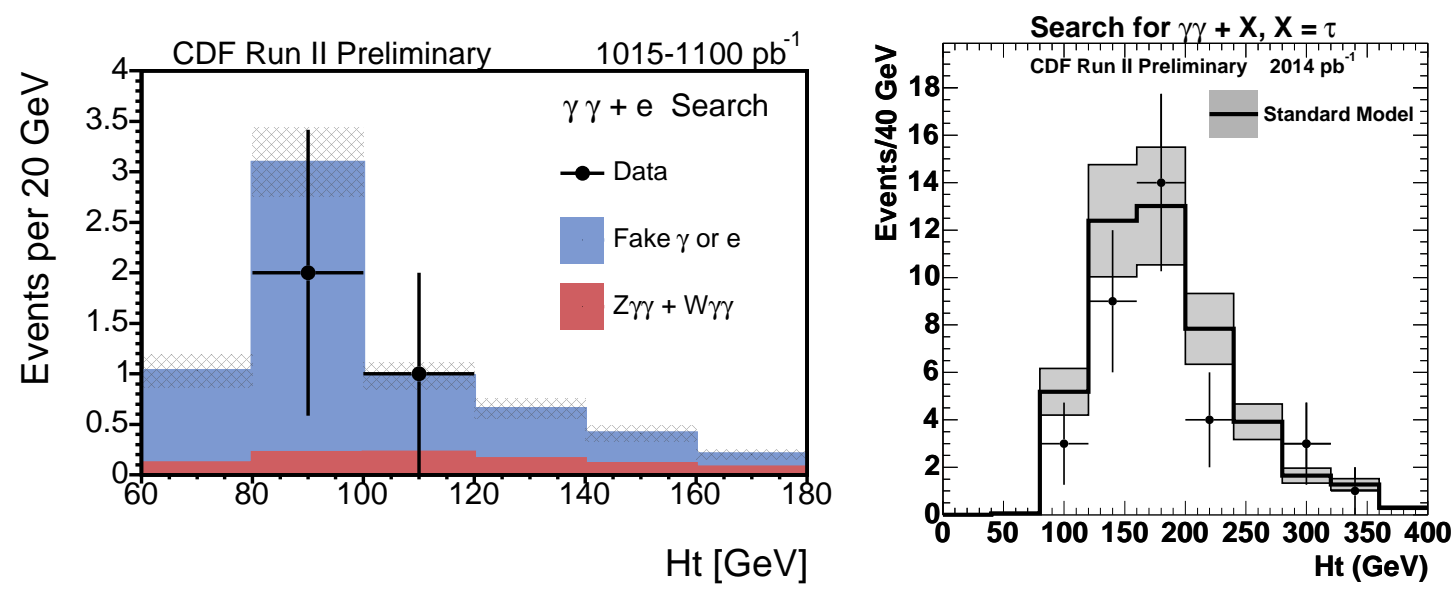

FIGURE 1. (left) The $H_{T}$ in the sample of two photons and an electron. (right)The $H_{T}$ in the sample of two photons and a tau.

We have also re-optimized the sample for the GMSB SUSY model[1]. In this model, all SUSY pair production proceeds through decays to two neutralinos, which each then decay to a photon and a gravitino, resulting in two photons, missing $E_{T}$ and other objects in every event. The optimization results in further cuts on $H_{T}$ (the scalar sum $E_{T}$ of all identified objects in the event) and the $\Delta \phi$ between the photons. The resulting limits, which include some sensitivity to the case when the neutralino is long-lived, are shown in Figure 2.

\section{SEARCH FOR PHOTONS, B-TAG AND MISSING $E_{T}$}

We select this sample in $2.0 \mathrm{fb}^{-1}$ of data collected by an inclusive isolated photon trigger. In the preliminary data selection, we require a photon in the central detector $\left(E_{T}>25 \mathrm{GeV}\right)$, and one jet $\left(E_{T}>15 \mathrm{GeV}\right)$ tagged with the standard secondary vertex algorithm, greatly enhancing the $b$-quark fraction. In the final event selection, we require a second jet, $\mathbb{E}_{T}>25 \mathrm{GeV}$, and $\mathbb{E}_{T}$ not parallel to any jet.

The backgrounds are a combination of real and misidentified photons and $b$-tags. Misidentified photons are primarily hadrons which decayed to photons. These events will create a shower shape that can be differentiated from single prompt photons if there is adequate statistics. The background will also convert to electron-positron pairs more often than single photons, and this difference in rate can be observed in the central 

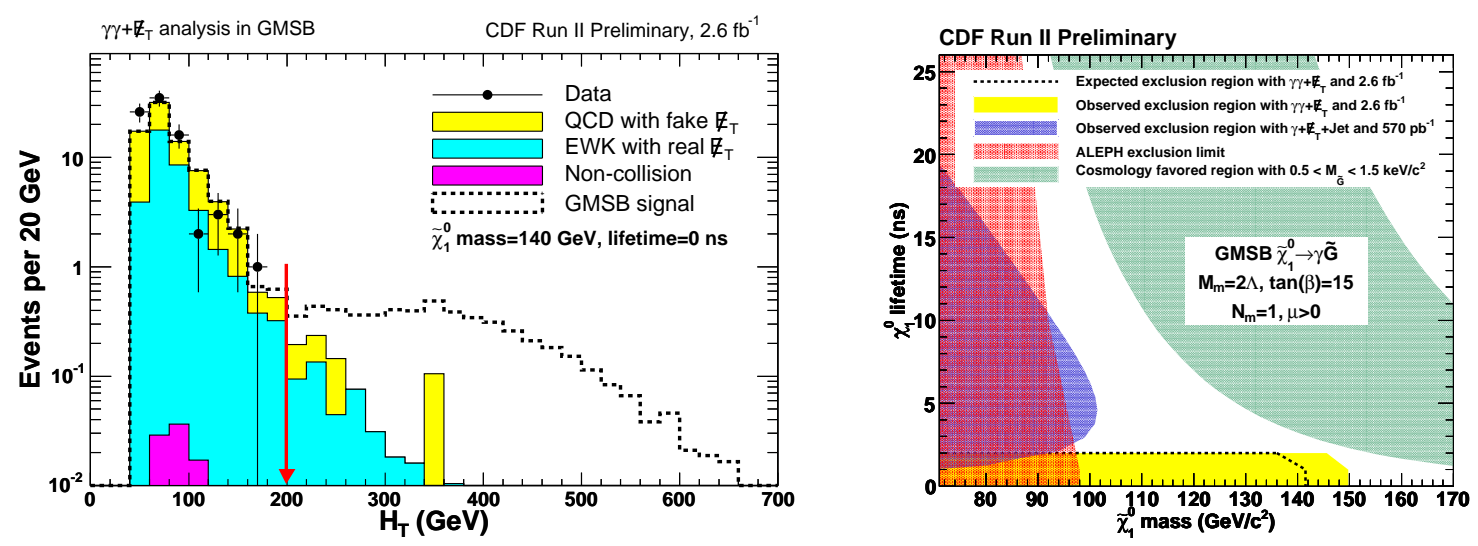

FIGURE 2. (left) The $H_{T}$ in the sample of two photons and missing $E_{T}$. (right) The limits on the GMSB model from the sample with two photons and missing $E_{T}$.

preshower detector. Using these two techniques we can estimate the background from misidentified photons.

In the case of a real photon and a fake $b$-tag, we can reverse the misidentified-photon procedure and also apply a jet-to- $b$-tag fake rate (derived from data) to the sample before $b$-tagging.

The final contribution to the SM background is real photons and real $b$-tags. This component is estimated from Monte Carlo, with normalization in data. The fraction of real $b$ tags in the preliminary data selection is determined by a fit to the mass of the tracks which are clustered into the secondary vertex in the $b$-tag. This mass is large for $b$-quarks, less for $c$ quarks, and even less for light quarks, allowing the discrimination. After this fraction is determined, the efficiency for the final cuts is measured in Monte Carlo and applied to arrive at the final estimate.

The final sample contains 617 events compared to a total SM expectation of $607 \pm 74 \pm 86$ events. Figure 3 shows two important kinematic distributions. This result is now published [2].
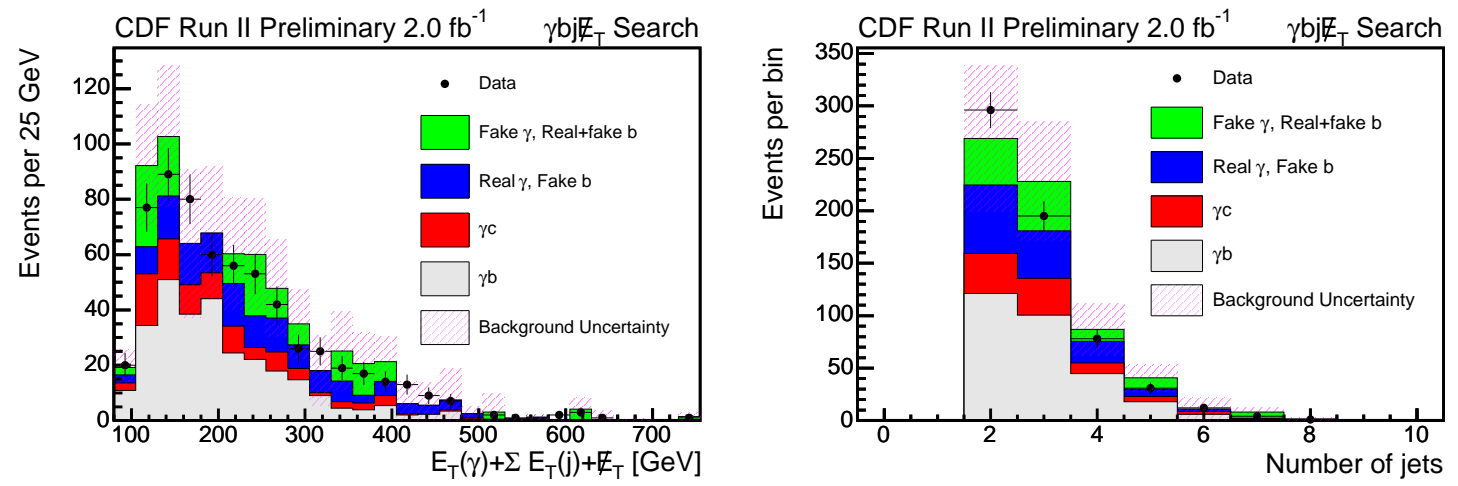

FIGURE 3. (left) The $H_{T}$ in the sample of a photon, a $b$-tagged jet,a second jet and missing $E_{T}$. (right) The number of jets in the sample. 


\section{SEARCH FOR FERMIOPHOBIC HIGGS}

The SM contains one Higgs doublet, but can be extended to two Higgs doublets. With the addition of some fine tuning, the lightest Higgs will decay only to boson pairs. Until the $W W$ branching fraction turns on around a Higgs mass of $100 \mathrm{GeV}$, the branching ratio to diphotons is nearly $100 \%$. With the gluon fusion diagram suppressed, most events would come from associated production $(W H)$.

We extract a diphoton sample from $3.0 \mathrm{fb}^{-1}$ of diphoton triggered events. We require two photons with $E_{T}>15 \mathrm{GeV}$. We accept events with both photons in the central detector, or one central and one in the forward detector $(2.2<|\eta|<2.8)$. Since associated production dominates, several approaches to optimization are possible. Many combinations of requirements which would select evidence of the $W$ or $Z$ boson were tried, but none were more effective than the requirement that the $P_{T}$ of the diphoton pair is greater than $75 \mathrm{GeV}$.

To establish the level of signal and background, we fit the diphoton mass spectrum to a background shape (a combination of exponentials and polynomials) and the signal shape from a Monte Carlo event sample. The limit as a function of Higgs mass is shown in Figure 4. The final mass limit on this type of Higgs boson is $106 \mathrm{GeV}$. This result is now published [3].
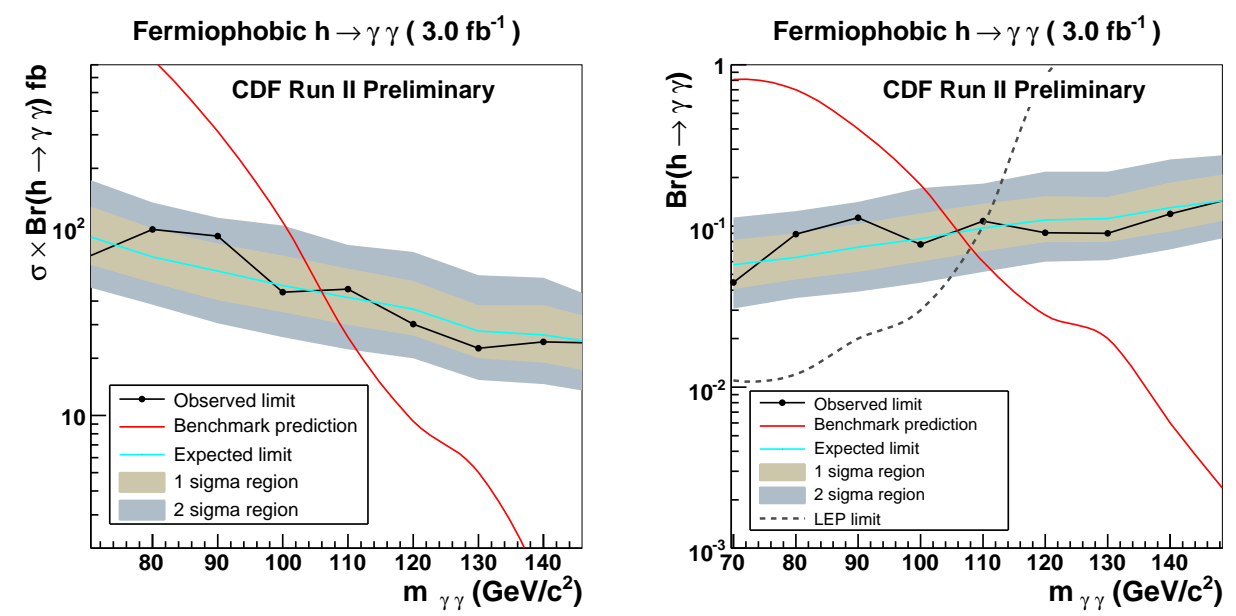

FIGURE 4. Limits on the Fermiophobic Higgs from the diphoton sample.

\section{REFERENCES}

1. B. C. Allanach et al. Eur. Phys. J C25 113 (2002); E. Blatz et al. J. High Energy Phys. 0305, 067 (2003).

2. T. Aaltonen et al., Phys. Rev. D80, 052003 (2009).

3. T. Aaltonen et al., Phys. Rev. Lett. 103, 061803 (2009). 